\title{
The Perioperative Surgical Home, Enhanced Recovery After Surgery and how integration of these models may improve care for medically complex patients
}

\author{
Tyrone G. Harrison, MD \\ Paul E. Ronksley, PhD \\ Matthew T. James, MD, PhD \\ Mary E. Brindle, MD, MPH \\ Shannon M. Ruzycki, MD, MPH \\ Michelle M. Graham, MD \\ Andrew D. McRae, MD, PhD \\ Kelly B. Zarnke, MD, MSc \\ Deirdre McCaughey, PhD, MBA \\ Chad G. Ball, MD, MSc \\ Elijah Dixon, MD, MSc \\ Brenda R. Hemmelgarn, MD, \\ $\mathrm{PhD}$
}

Accepted July 28, 2020

\author{
Correspondence to: \\ B. Hemmelgarn \\ Faculty of Medicine \& Dentistry \\ 2J2.01 Walter C. Mackenzie Health \\ Sciences Centre \\ University of Alberta \\ 8440112 St NW \\ Edmonton AB T6G 2R7 \\ Brenda.Hemmelgarn@ahs.ca
}

DOI: $10.1503 /$ cjs. 002020
Perioperative medicine is changing rapidly, and with this change comes the opportunity to improve upon current models of care delivery and integration within the health care system. Perioperative models of care are structured or conceptual arrangements for surgical patients before, during and after their surgery. Models of care such as the Perioperative Surgical Home and Enhanced Recovery After Surgery pathways are increasingly used to guide the structure of perioperative care delivery with an aim to improve patient outcomes and experience in Canadian settings. In this narrative review, we summarize the origins of these perioperative models of care. They are fundamentally different in scope and level of evidence. Both models have potential benefits and limitations to their broad implementation in our health care system. As currently developed, both models are limited in their application to patients with chronic disease. We discuss how these models of care can be used to develop integrated horizontal and vertical perioperative pathways in a Canadian setting. Such integration is a potential solution that will improve their applicability to patients with medically complex conditions and in times when health care systems are under pressure. We describe this approach using the example of patients with kidney failure receiving dialysis.

La médecine périopératoire évolue rapidement, ce qui est propice à l'amélioration des modèles de soins actuels et à leur intégration au système de santé. Les modèles de soins périopératoires sont des approches structurées ou conceptuelles à l'intention des patients de chirurgie avant, durant et après leur intervention. Les modèles de soins périopératoires, tels que les approches PSH (Perioperative Surgical Home) et ERAS (Enhanced Recovery After Surgery) sont de plus en plus utilisés pour orienter la structure et la prestation des soins périopératoires dans le but d'améliorer les résultats et l'expérience des patients dans les milieux de soins au Canada. Dans la présente synthèse narrative, nous retraçons l'origine de ces modèles de soins périopératoires. Ils sont fondamentalement différents aux plans de leur portée et de leur niveau de preuves. Mais leur déploiement à grande échelle dans nos systèmes de santé comporte des avantages et des inconvénients potentiels. Dans l'état actuel de leur développement, les 2 modèles ont une applicabilité limitée pour les patients atteints de maladies chroniques. Nous discutons de la façon dont ils peuvent être utilisés pour développer des approches horizontales et verticales intégrées au contexte canadien. Cette intégration est une solution envisageable qui améliorera leur application aux patients atteints de maladies complexes, à une époque où les systèmes de santé sont sous pression. Nous décrivons cette approche en prenant l'exemple des insuffisants rénaux dialysés.

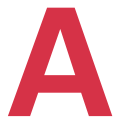

lthough the field of perioperative medicine is growing and evolving, a widely accepted current definition is the "integrated, multidisciplinary medical care of patients from the moment of contemplation of surgery until full recovery." ${ }^{1}$ As part of this evolving field, several integrated models and tools have been proposed to guide patients and their care providers through the perioperative period. In this narrative review, we discuss 2 of the most widely studied and implemented perioperative models of care, the Perioperative Surgical Home (PSH) and Enhanced Recovery After Surgery (ERAS). We explore the origins and key components of the 2 models, as well as their benefits and limitations. Finally, we present a proposed conceptual integration of these 2 models of care to manage surgical patients with chronic disease, including an example of how this could work in practice. 


\section{LITERATURE REVIEW}

In June 2019 and November 2019, we searched MEDLINE via Ovid for relevant full-text articles using Medical Subject Headings and text words with terms such as "perioperative care," "perioperative surgical home," "enhanced recovery" and "integrated care pathways," along with analogous terms. We also reviewed the reference lists of relevant articles for potential studies to be included.

\section{INTEGRATED PERIOPERATIVE PATHWAYS — PAST AND FUTURE}

Patients and care providers want similar outcomes from the perioperative experience: they desire optimal procedural success with minimal negative experiences and adverse events. It is in response to these shared perioperative goals that integrated care pathways have been introduced. These pathways address specific important components of a clinical problem and are framed around key tasks for patient care. ${ }^{2}$ In the perioperative setting, these pathways typically address preoperative risk assessment, optimization of modifiable risk factors and patient-specific care plans throughout the surgical journey. ${ }^{3}$ How these pathways are engineered is the focus of recent perioperative research, with movement away from traditionally siloed perioperative care delivery and toward more integrated delivery of care. ${ }^{1,4-6}$ These integrated care pathways represent the location-specific process of care for surgical patients in that setting. Perioperative models of care such as the PSH and ERAS can be conceptualized as providing the structure to develop integrated perioperative pathways or protocols, which are then tailored to be built within the health system that delivers the care for surgical patients. This is further detailed below.

The traditional perioperative pathway structure typically begins with a referral from a care provider to a surgeon. Once the patient is seen and assessed by the surgeon, discussions about options occur between the patient and the surgical team, and surgical plans may be set in motion. This may include operating room booking and referral to preoperative assessment clinics based on medical complexity, at the surgeon's discretion. These preoperative assessment clinics are staffed by various perioperative disciplines depending on location and commonly include anesthesia, internal medicine and cardiology specialists. ${ }^{7,8}$ In these clinics, perioperative medical risks (such as the risk of cardiac events or death) are discussed with the patient, often weeks or months after the surgical plans have been put in place. It is possible that the separation in time and space between discussions of surgical planning and medical risks, when too long to modify surgical plans, may introduce a barrier to optimal communication and perioperative care. Furthermore, separation of the discussion of surgical risks and benefits, and the discussion of medical risks and benefits into separate clinic visits can impede the informed decision- making process. As the preoperative assessment clinic visit often occurs shortly before the surgical procedure, time may be insufficient for optimization of modifiable risk factors. Within these noted disadvantages, there lies opportunity.

In the last decade, there has been considerable focus on restructuring the perioperative process. In contrast to the traditional approach, which prioritizes efficiency of care within each care silo, novel approaches to multidisciplinary collaboration in perioperative care that prioritize quality of care are increasingly being explored as viable options. ${ }^{1,4-7}$ For example, in these restructured pathways, patients may be assessed by surgical care providers and medical perioperative specialists during the same clinical encounter. This may allow for surgical options and medical risks associated with potential surgery or nonsurgical alternatives to be discussed with the patient and all members of the multidisciplinary team. ${ }^{1}$ If surgery is pursued, this can be preceded by adequate education, optimization of modifiable risk factors and prehabilitation. Prehabilitation in this setting refers to the perioperative processes of care that target patient exercise, nutrition, behaviours and mental health. ${ }^{9,10}$ Risk-stratified approaches to care could be designed and applied, such that patients at highest risk (i.e., for perioperative cardiac events or infections) could be triaged and managed in different and possibly more intensive ways than patients at lower risk. Furthermore, patients at low risk for perioperative complications may not need to see the entire perioperative team, which might lighten the clinical workload for perioperative care teams and the cost to finance such models.

In the traditional perioperative structure, limited time between preoperative medical consultation and surgery restricts the potential benefits of preoperative optimization. Times of health care system duress such as the COVID-19 pandemic place stress on all aspects of delivery of health care services, including surgical and perioperative services, which generally must continue in some magnitude. The efficiency and quality of perioperative care, from the preoperative optimization phase to the postoperative recovery phase, are sensitive to major health care changes such as the pandemic. Integrated perioperative care pathways, if implemented as discussed in this review, provide an opportunity to safely organize essential surgical services in an efficient way, and provide the framework for communication among perioperative multidisciplinary team members.

Perioperative pathway redesign offers opportunities to evaluate how patient-centred perioperative initiatives are best developed and delivered. It is within this evolution in perioperative medicine that initiatives such as the PSH and ERAS have been developed.

\section{The Perioperative Surgical Home}

\section{Origins}

The PSH was proposed by the American Society of Anesthesiologists (ASA) as an optimal patient-centred model of 
perioperative care in which patient health and access to appropriate surgical care can be optimized while the cost of care is reduced. ${ }^{11}$ It was developed to be analogous to, and integrate with, the Patient Centred Medical Home $(\mathrm{PCMH})$, which is the primary care model in which one health care provider team (i.e., the medical home) coordinates patient care and engages the greater network of care providers in a patient-centred manner. ${ }^{12}$ The PCMH is associated with improved patient satisfaction and quality of care, meeting the objectives the ASA established when the PSH was proposed. ${ }^{11,13}$

The PSH was developed as a model to encompass the entire perioperative period, from contemplation of surgery to postoperative recovery, after which the patient is returned to the care of his or her PCMH. If the transition from PSH to PCMH is seamless, with continuous care shared between the 2 entities and compliance with the pathway maintained, the benefits of the PSH are realized in reduced emergency department visits and hospital admissions for avoidable complications. ${ }^{12}$ The principles of this shared accountability are illustrated in initiatives such as the 2010 US Affordable Care Act, where shared accountability for health care quality among providers within accountable care organizations provided a foundation for $\mathrm{PCMH}$ and PSH development. ${ }^{13}$

\section{Elements}

The PSH can be conceptualized as the organizational umbrella under which all perioperative aspects of a patient's care are managed. ${ }^{20}$ The ASA identifies the strategic principles of the $\mathrm{PSH}$ as the following: ${ }^{11}$

- Patient-centred, with an emphasis on shared decisionmaking and patient engagement

- Physician-led

- Team-based, with multidisciplinary perioperative members

- Evidence-based, to reduce the variability in perioperative care

- Coordinated delivery of care

- Safe[ty]

Examples of PSH preoperative elements include targeted prehabilitation programs, triaged attendance at riskspecific preassessment clinics, admission to hospital preoperatively through a centralized perioperative clinic, and development of individualized care plans that transfer from the outpatient setting to the inpatient surgical setting. ${ }^{11,14}$ In addition, the PSH involves coordination of multidisciplinary care in the immediate preoperative period to ensure adherence to individualized care plans. Examples of PSH intraoperative elements include system-based elements such as techniques to reduce operating room delay, integrated operating room scheduling and electronic health records that bridge outpatient to inpatient care. Clinical intraoperative elements include goal-directed fluid administration, and management of pain and of anemia and bleeding. ${ }^{13,14}$ Finally, PSH postoperative elements include monitoring and continuation of initiatives started in other perioperative phases, early mobilization, education protocols for patients and caregivers, and incorporation of other "fast-track" surgery recommendations. However, all these elements are not necessary in every PSH model, as each is tailored to its local setting.

\section{Evidence}

Given the variability of content and implementation of PSH programs, consistent evidence of effectiveness as a model of perioperative care delivery is lacking. Kash and colleagues $^{11,13}$ reviewed whether pre-, intra- and postoperative PSH elements improved cost and efficiency outcomes or clinical outcomes. They found that cost and efficiency outcomes were significantly improved in $75 \%-88 \%$ of PSH studies and that clinical outcomes were significantly improved in $80 \%-90 \%$ of PSH studies. These results were mostly from observational studies and thus are likely limited by publication bias. Although not assessed formally, there was significant heterogeneity among the studies and outcomes that were included. Given these limitations, some PSH experts have asserted that there is a paucity of strong evidence that PSH implementation leads to improved patient, care provider and health care system outcomes. ${ }^{7}$

Optimal patient-centred study designs to investigate PSH models of care have been discussed, and PSH leaders have proposed research approaches to improve the PSH evidence base. ${ }^{714}$ To enhance patient-centred care of the surgical patient, development of context-specific, patientprioritized outcomes for these PSH evaluation studies are necessary. Outcomes would be optimally assessed with the use of randomized trial designs. Given the intensive financial and human resources necessary for PSH implementation, evaluation of the program or components of the program would be most suitably done with a pragmatic trial design such as a cluster-randomized trial (with implementing hospitals as a cluster) or a stepped-wedge clusterrandomized trial. Adequate evaluation of the PSH would require consultation with implementation scientists and a formal study of implementation, in addition to the study of cost and efficiency outcomes, clinical outcomes and patient-centred outcomes, in line with aspects of the Institute for Healthcare Improvement's triple aim. ${ }^{15}$ With this in mind, the ASA has committed financial support for a multihospital collaborative PSH implementation study (results pending). ${ }^{7,16}$

\section{Limitations}

Although there is a strong rationale for perioperative models of care such as the PSH, several limitations and barriers to the performance of the PSH have been identified. First, there are conflicting views concerning the exact role and scope of the PSH..$^{17,18}$ Critics of the PSH have challenged the model as being too nebulous to add value, and point to 
the necessity of a defined scope of the PSH and clear metrics by which it will be evaluated. ${ }^{19}$ These concerns are largely voiced by care providers who view the PSH as an "all-or-none" fixed organizational structure that is not amenable to site-specific modifications. ${ }^{17,18}$ However, the PSH is more effectively thought of as an umbrella under which institutional perioperative practices can be "strategically and operationally positioned." ${ }^{18}$ For example, if a clinical site had already implemented a colorectal surgery pathway, it could still be maintained within the new PSH structure. However, it would be implemented in a coordinated fashion alongside other perioperative strategies that are important within the local PSH model of care (i.e., integration within local health records or alongside other care pathways such as anemia/bleeding management or other surgery-specific pathways). ${ }^{18}$

Even so, the barriers to PSH acceptance and operationalization may prevent uptake. Although reduction of duplicate strategies within pathways may result in improved cost-efficiency, there are potential fiscal barriers. Most of the prohibitive costs are associated with design, implementation and assessment of PSH function within a local perioperative setting. ${ }^{7}$ In the US, where the majority of PSH literature is based, PSH implementation has been limited in settings where care providers are compensated based on fee-for-service models, as there is no financial incentive for front-line perioperative care providers to support PSH implementation. ${ }^{7}$ Until hospitals or health care systems recognize the potential cost benefits of a streamlined, patientcentred model for perioperative care and support the costs of the PSH, it will be difficult to persuade decision-makers that the PSH is worthwhile. ${ }^{7}$ Although there are a number of barriers to the widespread adoption of the PSH, with rigorous evaluation of PSH models and financial support from the ASA, these barriers are not insurmountable.

\section{Enhanced Recovery After Surgery}

\section{Origins}

Enhanced Recovery After Surgery is a perioperative model of care that is used to improve the perioperative experience and outcomes of surgical patients by means of standardized, evidence-based, surgery-specific protocols. The ERAS model had its origins in Denmark in the 1990s, when Dr. Henrik Kehlet, a colorectal surgeon, published his findings of significantly decreased time to discharge without increased postoperative complications after colonic resection procedures when a multimodal protocol was introduced in the perioperative period. ${ }^{20-22}$ Shortly thereafter, colleagues of Kehlet's from northern Europe formulated guidelines for the clinical care of patients undergoing colorectal surgery. ${ }^{23}$ This strategy and approach to perioperative clinical care formed the basis of the ERAS Society (http://erassociety.org/), which has the overall goal of developing evidence-based multimodal recommendations and guidelines for various surgical procedures or surgical patient populations to improve international perioperative outcomes and achieve early recovery. ${ }^{20,24}$

\section{Elements}

Enhanced Recovery After Surgery guidelines have been developed for numerous surgical procedures (or procedure groups) such as cardiac surgery, gynecologic cancer surgery, colorectal surgery, lung surgery, esophagectomy, breast reconstruction, head and neck cancer surgery, liver surgery, bariatric surgery, rectal/pelvic surgery, pancreaticoduodenectomy, cystectomy, gastrectomy and gastrointestinal surgery. ${ }^{25}$ In contrast, comparable perioperative guidelines for specific surgical patient populations (e.g., medically complex groups such as patients with kidney failure receiving dialysis) have not yet been developed. Each of these procedure-based guidelines identify specific evidence-based elements throughout the entirety of the perioperative period. These elements are determined through consensus and are graded based on the quality of the evidence and the strength of the recommendation for each specific guideline item. ${ }^{21}$ The ERAS Society recently published recommendations for guideline development in order to standardize this process and to provide a method of judging the quality of an ERAS Society guideline. ${ }^{26}$ To demonstrate the variety of items that are commonly included in ERAS guidelines, the elements found in the 2018 recommendations for elective colorectal surgery are summarized in Table $1 .{ }^{27}$

\section{Evidence}

As part of consensus determination with each ERAS Society guideline, recommendations are made only after the rigour of the available evidence is evaluated. ${ }^{26}$ Along with each ERAS protocol element, the grade of the recommendation (weak, strong) and quality of evidence (low, moderate, high) are summarized. ${ }^{21,26}$ Thus, ERAS has the additional advantage of having a more rigorous process of summarizing supporting evidence compared to other models of care such as the PSH. Enhanced Recovery After Surgery protocols are usually implemented without substantial local adaptation. This allows for greater generalizability of studies investigating ERAS effectiveness and greatly simplifies the evaluation of ERAS implementation, especially when compared to the PSH.

Length of stay has been found to be significantly reduced with several ERAS protocols. ${ }^{21}$ Postoperative complications have also been reported to be significantly reduced with ERAS protocol implementation. A metaanalysis of randomized trials investigating colorectal surgery after ERAS implementation showed that rates of some complications could be reduced by half when ERAS elements were followed. ${ }^{28}$ Moreover, when fidelity to ERAS was higher, postoperative complication rates were reduced proportionally. ${ }^{21}$ 
Table 1. Summary of 2018 ERAS Society protocol elements and grade of recommendation for elective colorectal surgery ${ }^{27}$

Grade of

Element recommendation

\section{Preadmission}

Information, education, counselling Strong

Preoperative optimization (risk assessment, Strong

smoking and alcohol cessation)

Prehabilitation Weak

Preoperative nutritional care (screening and Strong nutrition itself)

Management of anemia (targets, interventions) Strong

\section{Preoperative}

Prevention of nausea and vomiting Strong

Preanesthetic medication (anxiolysis and pain) Strong

Antimicrobial prophylaxis and skin preparation Intravenous antibiotic treatment,

chlorhexidine: strong

Oral antibiotic treatment, advanced skin decontamination: weak

Mechanical bowel preparation Strong

Preoperative fluid and electrolyte therapy to

maintain euvolemia

Preoperative fasting and carbohydrate loading Strong up to $2 \mathrm{~h}$ before surgery

Intraoperative

Standard Anesthetic Protocol Strong

Intraoperative fluid and electrolyte therapy Strong

aimed at near-zero fluid balance; goal-directed

fluid therapy in patients at high risk

Prevention of intraoperative hypothermia Strong

Minimally invasive surgical approach (compared Strong

to open surgery)

Avoidance of routine use of pelvic and Strong

peritoneal drains

\section{Postoperative}

Avoidance of nasogastric intubation; if placed, Strong

removal before reversal of anesthesia

Avoidance of opioids; use of NSAIDs; apply Strong

multimodal analgesia in combination with

spinal/epidural or blocks when indicated

Thromboprophylaxis: mechanical prophylaxis

until discharge; low-molecular-weight heparin

until 28 d postoperatively

Fluid and electrolyte administration aimed at

near-zero fluid balance, with avoidance of

isotonic crystalloids if giving fluid

Bladder catheterization for 1-3 d acceptable;

catheter should be removed routinely after this

period

Prevention of postoperative ileus through

multimodal strategy

Postoperative avoidance of hyperglycemia with

insulin therapy and stress-reducing techniques

Strong

Strong

Strong

Strong

Strong

weak on ward

Offering of food on day of surgery;
immunonutrition (anti-inflammatory

supplements such as L-arginine) to

malnourished patients

Early mobilization through education and patient Strong

encouragement

$I C U=$ intensive care unit; NSAID = nonsteroidal anti-inflammatory drug.
There are also financial incentives associated with ERAS implementation. In 2012, the UK National Health Service estimated that ERAS implementation had saved about $£ 275$ (roughly $\$ 440$ in 2012 Canadian dollars) per patient undergoing musculoskeletal surgical procedures, mostly through reduction in length of stay..$^{29}$ Across the implementation of several national ERAS protocols, about 140000 200000 bed days per year had been saved..$^{29}$ In Alberta, after initial implementation of the ERAS protocol for elective colorectal surgery, the median length of hospital stay decreased from 6.0 to 4.5 days, the 30 -day readmission risk decreased, and cost savings per patient ranged from US\$2806 to US\$5898 (Can\$3301 to Can\$6939). ${ }^{30}$ In a recent meta-analysis of 42 randomized trials of all types of surgery, ERAS programs were similarly associated with decreased length of stay, total complication rates and costs. ${ }^{31}$

\section{Limitations}

Although the evidence shows important benefits of ERAS protocols, considerable limitations remain. First, ERAS guidelines have not been developed for all surgical procedures. There are common elements across ERAS protocols, but the applicability or generalizability across surgical procedures is unknown. Likewise, the expected benefits of using items from an ERAS protocol for a different surgery type are unknown. There is a delay between publication of new perioperative strategies and incorporation of these strategies into ERAS guidelines; the lead author for each ERAS guideline updates the evidence base only every $2-3$ years. ${ }^{26}$ In addition, even after ERAS guidelines incorporate new evidence, full implementation requires health care systems to adopt and disseminate these changes within large, often inflexible, structures. The feasibility of implementing ERAS guidelines does not receive much consideration when new elements are incorporated into ERAS protocols.

Although the ERAS Society recognizes that optimization of chronic disease management is important, the ERAS guidelines rely heavily on reducing variation and standardizing care. ${ }^{21}$ These concepts are antithetical to the needs of surgical patients with complex chronic medical conditions, who require thoughtful, multidisciplinary adaptation of ERAS elements. As an example, several recommended practices could be harmful to patients with chronic heart failure or kidney disease (Table 1). Elements of fluid administration, anemia management and pain management (including the use of nonsteroidal anti-inflammatory drugs) may all be detrimental in these patient populations. ${ }^{32}$

\section{INTEGRATION OF THE PSH AND ERAS TO IMPROVE PERIOPERATIVE CARE DELIVERY FOR PATIENTS WITH CHRONIC DISEASE}

As both the PSH and ERAS models of care have been widely introduced in perioperative clinical environments, it is important to understand how they may be integrated in 
the same health care environment. The PSH structure is not fixed or specific to 1 type of surgery, and is tailored to specific perioperative institutions (hospitals and perioperative clinics). Generally, the services provided by all surgical specialties in a PSH health care setting should be compatible with the PSH structure. In contrast, with ERAS, protocols are designed to be specific to individual procedures or groups of similar procedures. The different structure and scope of the PSH and ERAS models of care allow them to integrate synergistically. The lack of evidence for the PSH can be balanced by the more rigorously evaluated ERAS protocols. Enhanced Recovery After Surgery protocols for different surgery types can be housed and supported through the PSH organizational structure. ${ }^{9,20}$ Development and implementation of ERAS protocols can be facilitated by the PSH, as many of the key institutional stakeholders will be embedded within the multidisciplinary PSH model of care..$^{20}$ Furthermore, collaboration among surgical and other specialties can be fostered under the PSH umbrella. This interaction between the PSH and ERAS can be conceptualized as intersecting vertical and horizontal pathways.

Cannesson and Mahajan ${ }^{33}$ made connections between the business environment and the perioperative environment with an analogy of vertical and horizontal pathway integration. In a business context, vertical integration strategies refer to situations in which companies acquire and control all aspects of a product, from development to front-line sales, to integrate themselves in their respective industry; Cannesson and Mahajan ${ }^{33}$ described the model of Apple as an example. Horizontal integration strategies are used by companies when they acquire other companies with similar product or service scope, with the aim of reducing competition. An example of this would be the methods used by the Walt Disney Company, which acquired Pixar Animation Studios, the Marvel Cinematic Universe and Lucasfilm to control the competition and claim a greater stake in the entertainment market. ${ }^{33}$ These concepts can be applied to the perioperative setting, where perioperative vertical pathways intersect with horizontal pathways. ${ }^{33}$ Enhanced Recovery After Surgery protocols are surgery specific and address multimodal perioperative elements for that surgical procedure alone. In this sense, ERAS protocols can be conceptualized as vertical pathways.

\section{Benefit of integrated perioperative pathways in complex surgical populations}

As described, challenges arise when standardized perioperative strategies for specific surgical procedures are applied to patients with chronic health conditions. It is with these chronic conditions that perioperative recommendations that are specific to the conditions themselves can be integrated as horizontal pathways. This is increasingly relevant to perioperative care because the average age of the surgical patient is increasing in the developed world, and at a faster rate than the average age of the general population. ${ }^{34}$ Along with this increased age comes an increased proportion of medical comorbidities, meaning that the overall surgical population is increasingly complex..$^{34,35}$ This complexity leads to interacting perioperative considerations and required adaptation of the perioperative process for individual patients.

Perioperative models are needed to develop protocols that accommodate these interacting chronic diseases; adapting evidence-based perioperative protocols may better improve postoperative outcomes in these patients. For example, patients with only 1 comorbid disease such as chronic heart failure may have a separate horizontal pathway where heart-failure-specific recommendations on anemia and bleeding management, medication reconciliation in the perioperative period or fluid administration could be housed. These recommendations would likely apply across many different surgical types or ERAS protocols. If the patient also has diabetes, a perioperative diabetes protocol could be cointegrated as a horizontal pathway alongside the heart failure pathway and the surgery-specific pathway. This example of a lattice of intersecting perioperative pathways may address the limitations of standardized ERAS protocols when used in isolation. The PSH as an organizational umbrella is well situated to accommodate both disease-specific horizontal pathways and ERAS vertical pathways.

\section{Perioperative considerations for people with kidney failure receiving dialysis}

Patients with kidney failure who are managed with dialysis often have multiple comorbidities and receive unique elements of care and, as a result, have unique challenges in the perioperative period. ${ }^{36,37}$ We briefly describe 3 such examples.

First, goal-directed fluid therapy is a challenge for people receiving dialysis, as both volume overload and hypotension caused by dialysis in the perioperative period are associated with poor postoperative outcomes. ${ }^{38-40}$ Optimal timing of dialysis, considering the balance between hemodialysis-related hypotension and potential volume overload, is even more challenging when inflexible, procedure-specific ERAS pathways are applied. Electrolyte management is more nuanced, as people receiving dialysis are often hyponatremic, hyperkalemic, hypocalcemic and acidemic, ${ }^{41}$ which may complicate perioperative management and timing of procedures.

As a second example, anemia is a common complication of chronic kidney disease that requires special consideration for patients undergoing surgery. Management of anemia for people receiving dialysis typically involves repletion and maintenance of iron stores with intravenous 
iron administration and administration of erythropoietinstimulating agents. ${ }^{42-44}$ Importantly, target hemoglobin ranges for these patients are not to a normal value; normalization of hemoglobin in patients with chronic kidney disease is associated with worse cardiovascular outcomes. ${ }^{45,46}$ However, preoperative anemia is a welldescribed risk factor for poor postoperative outcomes. ${ }^{47}$ To add complexity, people with kidney failure have a higher risk of bleeding perioperatively, and management of perioperative anticoagulation bridging requires an understanding of the renal clearance of anticoagulants to appropriately dose. ${ }^{48,49}$ Administration of blood products also has unique considerations for this population, as blood transfusions are immunogenic and may make it more difficult to find a suitable match for kidney transplantation. ${ }^{50}$ To our knowledge, there is currently no guideline recommendation for hemoglobin targets before major surgery for patients with kidney failure. ${ }^{43}$

Third, there are several surgery types that are uniquely common to this patient population (e.g., dialysis access surgery, kidney transplantation). These and other unique perioperative considerations for patients with chronic kidney disease are presented in Table 2 .

\section{PSH and ERAS integration for people with kidney failure receiving dialysis}

To show how the PSH and ERAS models of care can be integrated to improve the management of patients with kidney failure receiving dialysis around the time of surgery, we propose a conceptual model of care (Figure 1). As detailed above, PSH structure is not fixed and can be modified to suit local perioperative environments.

We can consider 2 main options for PSH design within a major Canadian hospital. The more ambitious endeavour involves a PSH that encompasses all perioperative care within the hospital for all patients and all surgical procedures (Figure 1A). The second option (perhaps implemented as a pilot model) would be a PSH specific to kidney disease (Figure 1B). Both models of perioperative care involve vertical surgery-specific pathways and existing ERAS pathways, and both would include horizontal diseasespecific pathways. These disease- and condition-specific pathways would include diseases that are not exclusive to patients with kidney disease but still commonly affect these patients (e.g., diabetes, cardiovascular disease), as well as those that are specific to this population (e.g., dialysis strategies, anemia of kidney disease management).

In addition, there is room for future development of ERAS protocols for surgical procedures that are unique to patients with kidney disease. For example, living and deceased kidney transplantation protocols, and protocols for dialysis access creation could be developed. These ERAS protocols could be organized under the PSH umbrella in either model as vertical pathways, intersecting
Table 2. Perioperative considerations for people with kidney failure receiving dialysis

\begin{tabular}{|c|c|}
\hline Consideration & Potential target for horizontal perioperative pathway \\
\hline $\begin{array}{l}\text { Volume and } \\
\text { hypotension } \\
\text { avoidance }\end{array}$ & $\begin{array}{l}\text { - Dialysis timing before and after surgery } \\
\text { - Volume management on dialysis } \\
\text { - Antihypertensive medication reconciliation in } \\
\text { perioperative period } \\
\text { - Avoidance of aggressive intravenous fluid } \\
\text { administration; adaptation to residual kidney function } \\
\text { and urine output }\end{array}$ \\
\hline $\begin{array}{l}\text { Electrolyte } \\
\text { derangements }\end{array}$ & $\begin{array}{l}\text { - Hyperkalemia } \\
\text { - Hyponatremia } \\
\text { - Hypocalcemia } \\
\text { - Metabolic acidosis } \\
\text { - Differences in management based on type of dialysis } \\
\text { modality }\end{array}$ \\
\hline Anemia & $\begin{array}{l}\text { - Complex iron-repletion strategies at baseline } \\
\text { - Patient likely taking erythropoietin-stimulating agents, } \\
\text { with unique adverse effects } \\
\text { - Lower hemoglobin targets than in the general } \\
\text { population } \\
\text { - For kidney transplantation candidates, blood } \\
\text { transfusions present risk of immune sensitization } \\
\text { (greater caution should be taken before blood } \\
\text { transfusions) }\end{array}$ \\
\hline Bleeding & $\begin{array}{l}\text { - Increased risk of perioperative bleeding } \\
\text { - Different pathophysiology of bleeding, necessitating } \\
\text { directed management of bleed risk } \\
\text { - Altered metabolism of anticoagulants }\end{array}$ \\
\hline Miscellaneous & $\begin{array}{l}\text { - Altered metabolism of opioid medications } \\
\text { - Lack of validated risk-stratification tools } \\
\text { - Higher risk of postoperative cardiac complications with } \\
\text { atypical symptoms, with events more challenging to } \\
\text { diagnose }\end{array}$ \\
\hline
\end{tabular}

with the disease- and condition-specific horizontal pathways. Development of a perioperative model that is purposefully organized for medically complex cohorts would also facilitate research on ERAS elements for adaptation of ERAS guidelines to these populations.

\section{Conclusion}

Perioperative medicine is evolving, and with this evolution there is opportunity to improve upon how this care is structured and delivered within health care systems. The PSH and ERAS are perioperative models of care that improve patients' perioperative experience and outcomes. Although the 2 models have several differences in purpose and scope, their integration as intersecting vertical and horizontal perioperative pathways may collectively address limitations faced with either model on its own. Collaboration among the multidisciplinary perioperative team members is key for such integration, and effective leadership to connect key stakeholders is necessary. With strong implementation and a clear strategy for evaluation, these perioperative models of care might be integrated effectively to address complex perioperative populations. 
A

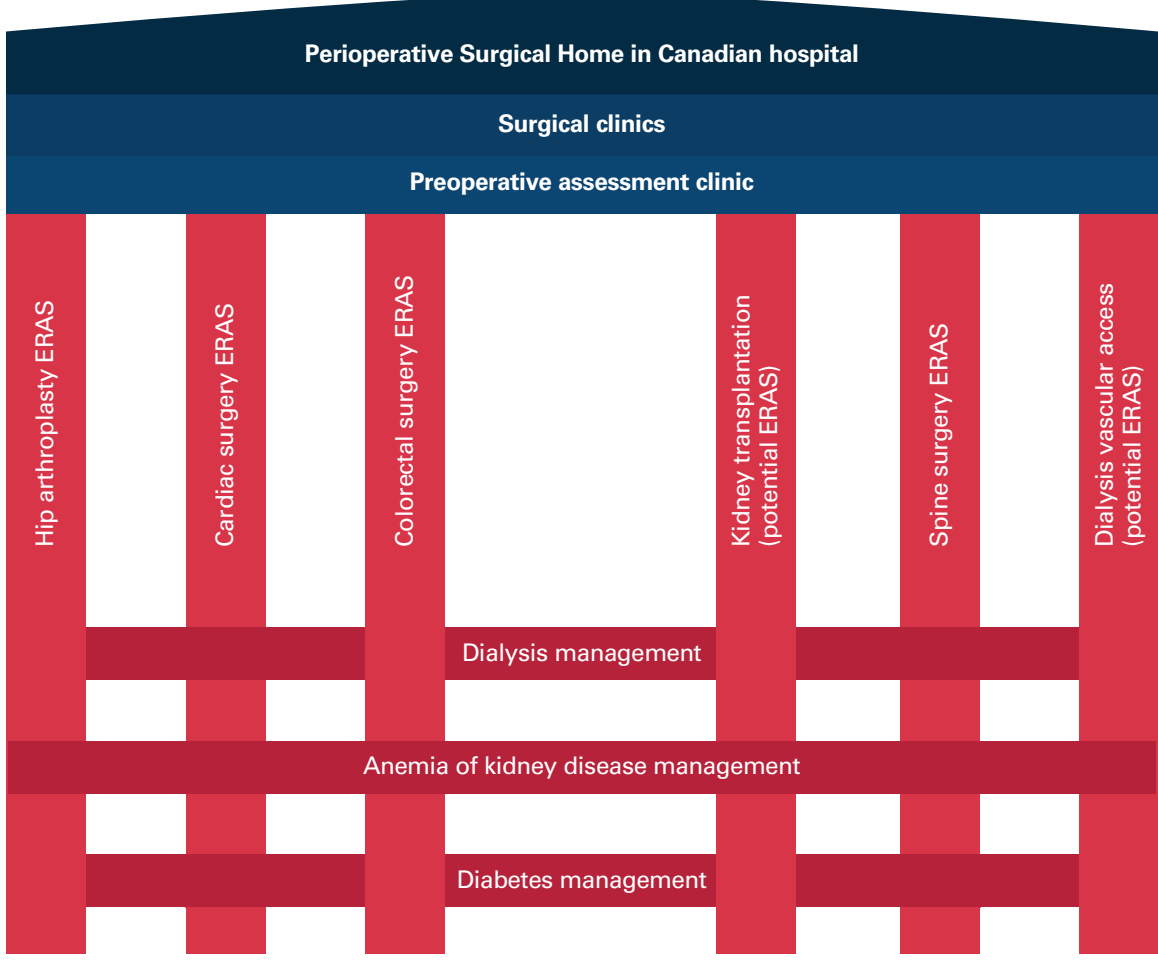

B

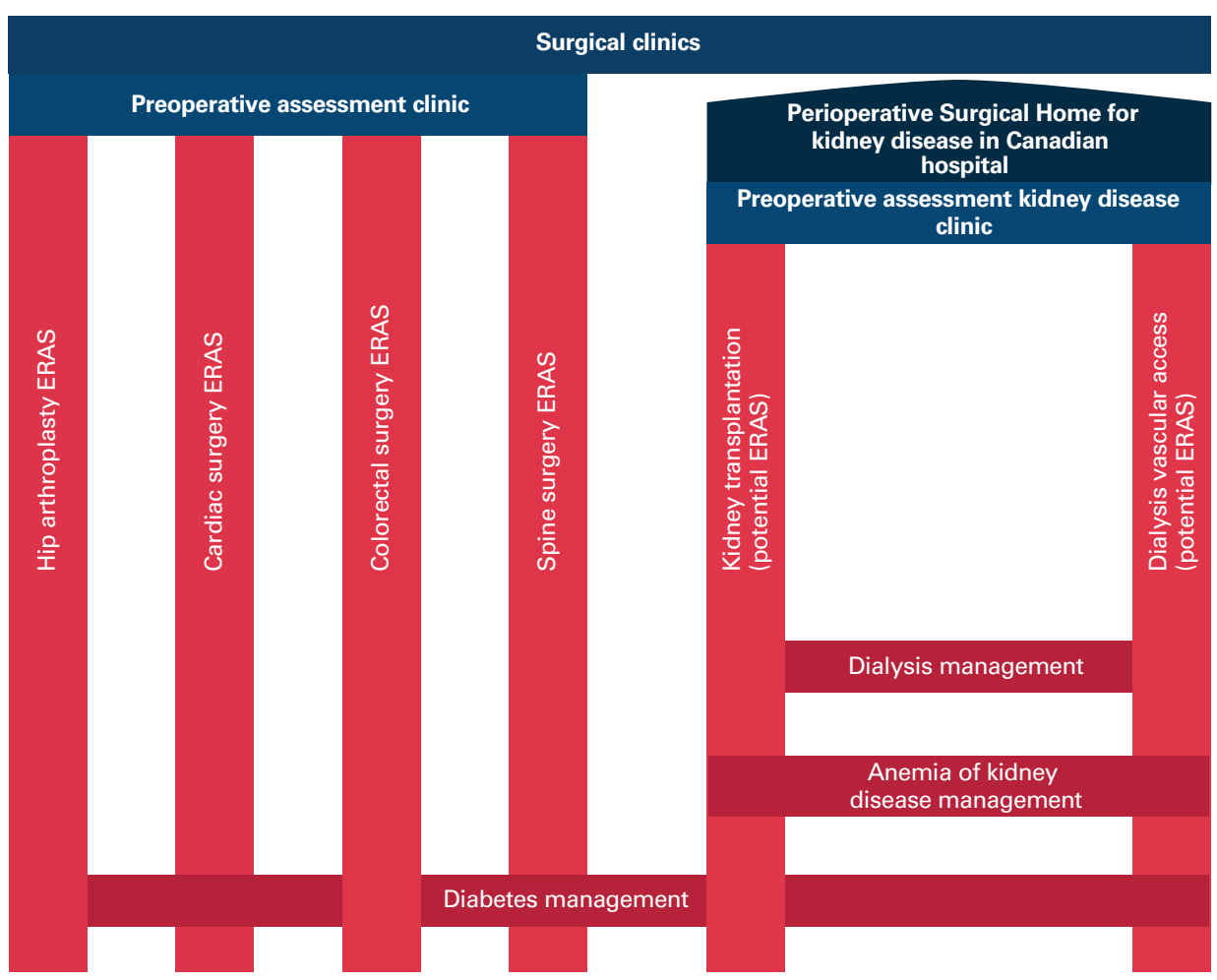

Fig. 1. Conceptual model of intersecting vertical and horizontal surgical pathways within a Canadian hospital setting, with a Perioperative Surgical Home that encompasses all surgical populations and procedures (A), and only patients with kidney failure and pathways specific to kidney disease (B). ERAS = Enhanced Recovery After Surgery. 
Affiliations: From the Department of Medicine, University of Calgary, Calgary, Alta. (Harrison, James, Ruzycki, Zarnke, Hemmelgarn); the Department of Community Health Sciences, University of Calgary, Calgary, Alta. (Harrison, James, Ronksley, Ruzycki, McRae, McCaughey, Dixon); the O'Brien Institute for Public Health, Cumming School of Medicine, University of Calgary, Calgary, Alta. (Ronksley, Zarnke, McCaughey, James); the Libin Cardiovascular Institute of Alberta, Cumming School of Medicine, University of Calgary, Calgary, Alta. (James); the Department of Surgery, University of Calgary, Calgary, Alta. (Brindle, Ball, Dixon); the Department of Emergency Medicine, University of Calgary, Calgary, Alta. (McRae); the Mazankowski Alberta Heart Institute, Edmonton, Alta. (Graham); and the Department of Medicine, University of Alberta, Edmonton, Alta. (Graham, Hemmelgarn).

Competing interests: Matthew James is the principal investigator of an investigator-initiated research grant from Amgen Canada, outside the submitted work. No other competing interests were declared.

Contributors: T. Harrison, P. Ronksley, M. James and B. Hemmelgarn designed the study. T. Harrison acquired the data (literature review), which T. Harrison, P. Ronksley, M. James, M. Brindle, S. Ruzycki, M. Graham, A. McRae, K. Zarnke, D. McCaughey, C. Ball, E. Dixon and B. Hemmelgarn analyzed. T. Harrison wrote the article, which P. Ronksley, M. James, S. Ruzycki, M. Brindle, M. Graham, A. McRae, K. Zarnke, D. McCaughey, C. Ball, E. Dixon and B. Hemmelgarn critically reviewed. All authors approved the article for publication.

Content licence: This is an Open Access article distributed in accordance with the terms of the Creative Commons Attribution (CC BYNC-ND 4.0) licence, which permits use, distribution and reproduction in any medium, provided that the original publication is properly cited, the use is noncommercial (i.e., research or educational use), and no modifications or adaptations are made. See: https://creativecommons. org/licenses/by-nc-nd/4.0/.

Disclaimer: Chad Ball is editor-in-chief and Mary Brindle is an associate editor of the Canadian Fournal of Surgery. Neither was involved in any aspect of the editorial review of this paper.

Funding: Tyrone Harrison is supported by a Kidney Research Scientist Core Education and National Training Program postdoctoral fellowship (cosponsored by the Kidney Foundation of Canada and Canadian Institutes of Health Research [CIHR]) and the Clinician Investigator Program at the University of Calgary. Paul Ronksley is supported by a CIHR Project grant. Mary Brindle is supported by the Alberta Children's Hospital Foundation MacNeill Chair in Pediatric Surgery. The funders had no role in study design, data collection, analysis or reporting, or the decision to submit for publication.

\section{References}

1. Grocott MPW, Edwards M, Mythen MG, et al. Peri-operative care pathways: re-engineering care to achieve the 'triple aim.' Anaesthesia 2019;74(Suppl 1):90-9.

2. Campbell H, Hotchkiss R, Bradshaw N, et al. Integrated care pathways. BM7 1998;316:133-7.

3. Grocott MP, Pearse RM. Perioperative medicine: The future of anaesthesia? Br 7 Anaesth 2012;108:723-6.

4. Grocott MPW, Plumb JOM, Edwards M, et al. Re-designing the pathway to surgery: better care and added value. Perioper Med (Lond) 2017;6:9.

5. Klein AA, Earnshaw JJ. Perioperative care and collaboration between surgeons and anaesthetists - it's about time. Br F Surg 2020;107:e6-7.

6. Dupree JM, Patel K, Singer SJ, et al. Attention to surgeons and surgical care is largely missing from early Medicare accountable care organizations. Health Aff (Millwood) 2014;33:972-9.

7. Kain $\mathrm{ZN}$, Vakharia S, Garson L, et al. The perioperative surgical home as a future perioperative practice model. Anesth Analg 2014; 118:1126-30.

8. Bader AM, Sweitzer B, Kumar A. Nuts and bolts of preoperative clinics: the view from three institutions. Cleve Clin 7 Med 2009;76 (Suppl 4):S104-11.
9. Paiste J, Simmons JW, Vetter TR. Enhanced Recovery After Surgery in the setting of the Perioperative Surgical Home. Int Anesthesiol Clin 2017;55:135-47.

10. Vetter TR, Boudreaux AM, Jones KA, et al. The perioperative surgical home: how anesthesiology can collaboratively achieve and leverage the triple aim in health care. Anesth Analg 2014;118:1131-6.

11. Kash B, Cline K, Menser T, et al. The Perioperative Surgical Home (PSH): a comprehensive literature review for the American Society of Anesthesiologists. 2014. Available: https://www.asahq.org/psh/ / media/sites/psh/files/pshlitreview.pdf (accessed 2019 Nov. 19).

12. Vetter TR, Goeddel LA, Boudreaux AM, et al. The Perioperative Surgical Home: How can it make the case so everyone wins? BMC Anesthesiol 2013;13:6.

13. Kash BA, Zhang Y, Cline KM, et al. The perioperative surgical home (PSH): a comprehensive review of US and non-US studies shows predominantly positive quality and cost outcomes. Milbank $Q$ 2014;92:796-821.

14. Vetter TR, Ivankova NV, Goeddel LA, et al. An analysis of methodologies that can be used to validate if a Perioperative Surgical Home improves the patient-centeredness, evidence-based practice, quality, safety, and value of patient care. Anesthesiology 2013;119:1261-74.

15. Berwick DM, Nolan TW, Whittington J. The triple aim: care, health, and cost. Health Aff (Millwood) 2008;27:759-69.

16. Duncan MJ. Perioperative Surgical Home, fixing a fragmented process to improve quality of care. Mo Med 2019;116:53-7.

17. Mariano ER, Vetter TR, Kain ZN. The Perioperative Surgical Home is not just a name. Anesth Analg 2017;125:1443-5.

18. Vetter TR. Perioperative Surgical Home models. Anesthesiol Clin 2018;36:677-87.

19. Soybel DI, Knuf K. The Perioperative Surgical Home: Cui bono? FAMA Surg 2016;151:1003-4.

20. Elhassan A, Elhassan I, Elhassan A, et al. Perioperative surgical home models and enhanced recovery after surgery. 7 Anaesthesiol Clin Pharmacol 2019;35(Suppl 1):S46-50.

21. Ljungqvist O, Scott M, Fearon KC. Enhanced Recovery After Surgery: a review. FAMA Surg 2017;152:292-8.

22. Kehlet H, Mogensen T. Hospital stay of 2 days after open sigmoidectomy with a multimodal rehabilitation programme. Br F Surg 1999; 86:227-30.

23. Fearon KC, Ljungqvist O, Von Meyenfeldt M, et al. Enhanced recovery after surgery: a consensus review of clinical care for patients undergoing colonic resection. Clin Nutr 2005;24:466-77.

24. Cannesson M, Kain Z. The perioperative surgical home: an innovative clinical care delivery model. 7 Clin Anesth 2015;27:185-7.

25. ERAS guidelines. Stockholm: ERAS Society. Available: www. erassociety.org/guidelines/list-of-guidelines/ (accessed 2019 Nov. 12).

26. Brindle M, Nelson G, Lobo DN, et al. Recommendations from the ERAS $®$ Society for standards for the development of enhanced recovery after surgery guidelines. B7S Open 2020;4:157-63.

27. Gustafsson UO, Scott MJ, Hubner M, et al. Guidelines for perioperative care in elective colorectal surgery: Enhanced Recovery After Surgery (ERAS®) Society recommendations: 2018. World F Surg 2019;43:659-95.

28. Greco M, Capretti G, Beretta L, et al. Enhanced recovery program in colorectal surgery: a meta-analysis of randomized controlled trials. World 7 Surg 2014;38:1531-41.

29. Enhanced Recovery Partnership. Fulfilling the potential: a better journey for patients and a better deal for the NHS. London (UK): NHS Improvement; 2012.

30. Nelson G, Kiyang LN, Crumley ET, et al. Implementation of Enhanced Recovery After Surgery (ERAS) across a provincial healthcare system: the ERAS Alberta colorectal surgery experience. World $\mathcal{F}$ Surg 2016;40:1092-103.

31. Lau CS, Chamberlain RS. Enhanced Recovery After Surgery programs improve patient outcomes and recovery: a meta-analysis. World F Surg 2017;41:899-913. 
32. Nasr R, Chilimuri S. Preoperative evaluation in patients with endstage renal disease and chronic kidney disease. Health Serv Insights 2017:10. doi.org/10.1177/1178632917713020.

33. Cannesson M, Mahajan A. Vertical and horizontal pathways: intersection and integration of Enhanced Recovery After Surgery and the Perioperative Surgical Home. Anesth Analg 2018;127:1275-7.

34. Fowler AJ, Abbott TEF, Prowle J, et al. Age of patients undergoing surgery. Br 7 Surg 2019;106:1012-8.

35. Johnston MC, Crilly M, Black C, et al. Defining and measuring multimorbidity: a systematic review of systematic reviews. Eur 7 Public Health 2019;29:182-9.

36. Harrison T, James MT, Zarnke KB, et al. Estimated GFR and incidence of major surgery: a population-based cohort study. Am 7 Kidney Dis 2021;77:365-75.e1.

37. Fraser SD, Taal MW. Multimorbidity in people with chronic kidney disease: implications for outcomes and treatment. Curr Opin Nephrol Hypertens 2016;25:465-72.

38. Sessler DI, Meyhoff CS, Zimmerman NM, et al. Period-dependent associations between hypotension during and for four days after noncardiac surgery and a composite of myocardial infarction and death: a substudy of the POISE-2 Trial. Anesthesiology 2018; 128:317-27.

39. Holte K, Sharrock NE, Kehlet H. Pathophysiology and clinical implications of perioperative fluid excess. Br 7 Anaesth 2002;89:622-32.

40. Deng J, Lenart J, Applegate RL. General anesthesia soon after dialysis may increase postoperative hypotension - a pilot study. Heart Lung Vessel 2014;6:52-9.
41. Dhondup T, Qian Q. Electrolyte and acid-base disorders in chronic kidney disease and end-stage kidney failure. Blood Purif 2017;43:179-88.

42. Madore F, White CT, Foley RN, et al. Clinical practice guidelines for assessment and management of iron deficiency. Kidney Int Suppl 2008;110:S7-11.

43. Moist LM, Foley RN, Barrett BJ, et al. Clinical practice guidelines for evidence-based use of erythropoietic-stimulating agents. Kidney Int Suppl 2008;110:S12-8.

44. White CT, Barrett BJ, Madore F, et al.; TREAT Investigators. Clinical practice guidelines for evaluation of anemia. Kidney Int Suppl 2008;110:S4-6.

45. Pfeffer MA, Burdmann EA, Chen CY, et al. A trial of darbepoetin alfa in type 2 diabetes and chronic kidney disease. $N$ Engl 7 Med 2009;361:2019-32.

46. Besarab A, Bolton WK, Browne JK, et al. The effects of normal as compared with low hematocrit values in patients with cardiac disease who are receiving hemodialysis and epoetin. NEngl f Med 1998;339:584-90.

47. Munoz M, Acheson AG, Auerbach M, et al. International consensus statement on the peri-operative management of anaemia and iron deficiency. Anaesthesia 2017;72:233-47.

48. Acedillo RR, Shah M, Devereaux PJ, et al. The risk of perioperative bleeding in patients with chronic kidney disease: a systematic review and meta-analysis. Ann Surg 2013;258:901-13.

49. Lai A, Davidson N, Galloway SW, et al. Perioperative management of patients on new oral anticoagulants. Br F Surg 2014;101:742-9.

50. Obrador GT, Macdougall IC. Effect of red cell transfusions on future kidney transplantation. Clin 7 Am Soc Nephrol 2013;8:852-60. 